\title{
Left ventricular ejection fraction... What else?
}

\author{
Marta Jiménez-Blanco ${ }^{1}$, David Cordero ${ }^{1,2}$, José Luis Zamorano ${ }^{1,2}$ \\ ${ }^{1}$ Department of Cardiology, University Hospital Ramon y Cajal, Madrid, Spain \\ ${ }^{2}$ Centro de Investigación Biomédica en Red en Enfermedades Cardiovasculares (CIBERCV), \\ Madrid, Spain
}

Article p. 97

The main terminology used to describe heart failure (HF) is based on the measurement of the left ventricular ejection fraction (LVEF), and current guidelines divide patients into three subgroups: heart

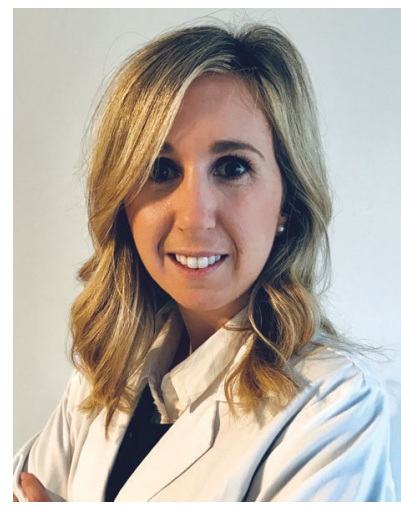
failure with preserved ejec-
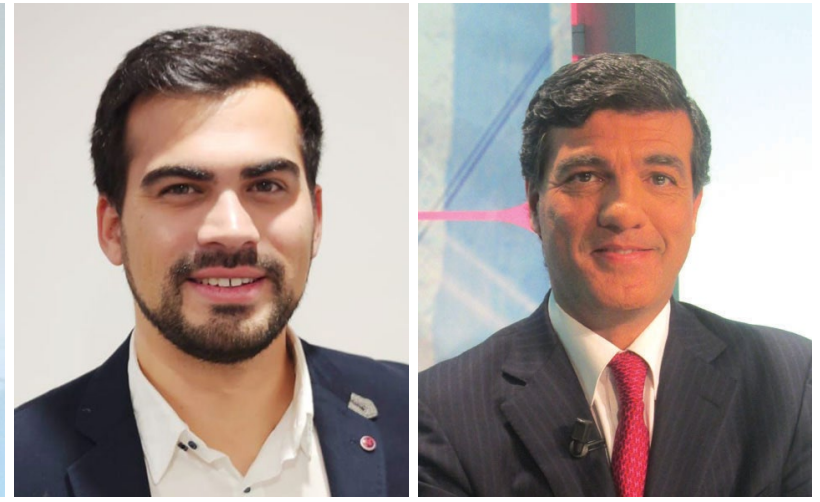

tion fraction (HFpEF) if LVEF > 50\%, heart failure with mid-range ejection fraction (HFmrEF) if LVEF is between $40 \%$ and $49 \%$ and heart failure with reduced ejection fraction (HFrEF) if LVEF is $<40 \%$ [1]. LVEF has been the main selection criteria for most clinical trials during the past three decades, however, improved morbidity and mortality rates have only been shown in patients with HFrEF.

On the contrary, despite a long history of clinical trials on $\mathrm{HFpEF}$, there has been no proof of any single drug which improves survival rates in this subset of patients. There is a common understanding that this lack of success could be linked to the fact that $\mathrm{HFpEF}$ is comprised of a wide variety of unrelated pathologies.

In this issue of 'Cardiology Journal', Junbo Ge proposes a new classification for $\mathrm{HFpEF}$ into five categories based on their etiology and pathophysiology: 1) vascular related HFpEF, 2) cardiomyopathy-related $\mathrm{HFpEF}, 3)$ right-heart and pulmonaryrelated $\mathrm{HFpEF}$, 4) valvular and rhythm related $\mathrm{HFpEF}$, and 5) extracardiac-disease related $\mathrm{HFpEF}$ [2]. This new categorization could potentially allow more targeted clinical trials, and hopefully achieve some benefit in certain subgroups.
However, the main problem may not be $\mathrm{HFpEF}$ itself, but by the way HF is diagnosed and classified. The concept of LVEF, which is defined by dividing the stroke volume by the end-diastolic volume, was developed in the 1960s [3], and has become a cornerstone in cardiology since then. Nonetheless, HF patients, disease phenotypes and technology have evolved significantly since then, and nowadays the information provided by the LVEF is inadequate or insufficient in many situations.

There are various setbacks to two-dimensional echocardiography measured LVEF [4]. First and foremost, there is a $10 \%$ of interobserver variability [5], which may lead to the same patient falling into different categories depending on the echocardiographer who reads the study, and it varies depending on the imaging method used and on the cut-planes. Secondly, it is widely influenced by geometry, which is particularly important in ischemic cardiomyopathy, and by ventricular loading, especially afterload. Moreover, it does not take into account the complexity of myocardial mechanics or the speed of contraction, and its assessment in patients with mechanical dyssynchrony, which 
is still under debate. Last, but not least, ejection fraction (EF) has proven prognostic significance in patients with $\mathrm{EF}<40 \%$, but in patients with $\mathrm{EF}>40 \%$ there is no correlation between higher $\mathrm{EF}$ and better outcomes [6].

Taking into account the abovementioned statements, certain doubts arise regarding the suitability of LVEF as the best method to classify HF patients. What if LVEF were too simple a classification for such a complex disease?

Changing the current classification would probably shake the foundations of $\mathrm{HF}$ as we know it today, since most clinical trials that have shown benefit were based on this concept. However, having said this, there is clearly room for improvement.

Left ventricular mechanics (as measured by two-dimensional speckle-tracking echocardiography), three-dimensional echocardiography or cardiac magnetic resonance (CMR) all allow for an improved assessment of myocardial function. Global longitudinal strain has been shown to correlate with prognosis in all patients with HF, regardless their LVEF, and provides the greatest incremental information when the LVEF is relatively preserved and regional wall motion scores are normal [7]. On the other hand, CMR plays an important role in the diagnosis of many HFpEF pathologies, such as hypertrophic cardiomyopathy or cardiac amyloidosis, and its role on prognosis in many others has already been stablished [8].

On top of that, CMR is the most reliable method to identify inflammation, which is the main histopathological substrate of many different causes of HFpEF. Sustained inflammation leads to interstitial fibrosis and myocardial hypertrophy, that cause impaired left ventricular relaxation and coronary microvascular dysfunction [9].

The point of view herein, is that it is not only necessary to identify the etiology of HF, as Junbo $\mathrm{Ge}$ [2] proposes in his article, but also to create new diagnostic and prognostic scores that include all the aforementioned tools, allowing a better classification of $\mathrm{HF}$ patients. It is highly probable that treatments based on integrating all this information will provide better clinical results than those based solely on LVEF. Recent drugs that have failed to prove benefit in $\mathrm{HFpEF}$ may have a role in a subset of patients with reduced left ventricular mechanics or active inflammation.

In conclusion, it seems that the actual concept of LVEF is set in stone, but perhaps it is time to think out of the box and rewrite the basics of heart failure.

\section{Conflict of interest: None declared}

\section{References}

1. Ponikowski P, Voors A, Anker S, et al. 2016 ESC Guidelines for the diagnosis and treatment of acute and chronic heart failure. Eur Heart J. 2016; 37(27): 2129-2200, doi: 10.1093/eurheartj/ehw128.

2. Ge J. Coding proposal on phenotyping heart failure with preserved ejection fraction: A practical tool for facilitating etiology-oriented therapy. Cardiol J. 2020; 27(1): 97-98, doi: 10.5603/ CJ.2020.0023.

3. Folse R, Braunwald E. Determination of fraction of left ventricular volume ejected per beat and of ventricular end-diastolic and residual volumes. Experimental and clinical observations with a precordial dilution technic. Circulation. 1962; 25: 674-685, doi: 10.1161/01.cir.25.4.674, indexed in Pubmed: 13893859.

4. Marwick T. Ejection fraction pros and cons. J Am Coll Cardiol. 2018; 72(19): 2360-2379, doi: 10.1016/j.jacc.2018.08.2162.

5. Nayyar S, Magalski A, Khumri TM, et al. Contrast administration reduces interobserver variability in determination of left ventricular ejection fraction in patients with left ventricular dysfunction and good baseline endocardial border delineation. Am J Cardiol. 2006; 98(8): 1110-1114, doi: 10.1016/j.amjcard.2006.05.038, indexed in Pubmed: 17027582.

6. Solomon SD, Anavekar N, Skali H, et al. Influence of ejection fraction on cardiovascular outcomes in a broad spectrum of heart failure patients. Circulation. 2005; 112(24): 3738-3744, doi: 10.1161/CIRCULATIONAHA.105.561423, indexed in Pubmed: 16330684.

7. Park JJ, Park JB, Park JH, et al. Global longitudinal strain to predict mortality in patients with acute heart failure. J Am Coll Cardiol. 2018; 71(18): 1947-1957, doi: 10.1016/j.jacc.2018.02.064, indexed in Pubmed: 29724346.

8. Raman B, Ariga R, Spartera M, et al. Progression of myocardial fibrosis in hypertrophic cardiomyopathy: mechanisms and clinical implications. Eur Heart J Cardiovasc Imaging. 2019; 20(2): 157-167, doi: 10.1093/ehjci/jey135, indexed in Pubmed: 30358845.

9. Loai S, Cheng HLM. Heart failure with preserved ejection fraction: the missing pieces in diagnostic imaging. Heart Fail Rev. 2019 [Epub ahead of print], doi: 10.1007/s10741-019-09836-8, indexed in Pubmed: 31364028. 